\title{
Hipersensibilidade a corticosteroides - Uma revisão
}

\section{Hypersensitivity to corticosteroids - A review}

Rev Port Imunoalergologia 2020;28 (3): I49-160

Iolanda Alen Coutinho, Joana Pita, Ana Luísa Moura, Marta Alves, Carlos Loureiro, Ana Todo Bom

Serviço de Imunoalergologia, Centro Hospitalar Universitário de Coimbra, Coimbra

\section{RESUMO}

Os corticosteroides são fármacos anti-inflamatórios amplamente utilizados na prática clínica e as reações de hipersensibilidade, com apresentação imediata ou tardia têm sido cada vez mais descritas na literatura. As reações de hipersensibilidade podem ocorrer devido aos corticosteroides ou aos excipientes utilizados nas suas preparações. Os testes cutâneos podem auxiliar na confirmação diagnóstica e na identificação de uma alternativa terapêutica.

O presente trabalho tem como objetivo a revisão do conhecimento atual sobre a apresentação clínica, a avaliação e abordagem diagnóstica das reações de hipersensibilidade imediata e tardia a corticosteroides.

Palavras-chave: Corticosteroides, hipersensibilidade, testes cutâneos.

\section{ABSTRACT}

Corticosteroids are anti-inflammatory drugs widely used in clinical practice. Hypersensitivity reactions, with immediate or late presentation, have been increasingly described in the literature. Hypersensitivity reactions may occur due to the corticosteroid or due to the excipients used in its preparation. Skin tests may aid in the diagnostic confirmation and in the identification of an alternative therapeutic agent.

Our objective is to review current knowledge about the clinical presentation, evaluation and diagnostic approach of immediate and delayed hypersensitivity reactions to corticosteroids.

Key-words: Corticosteroids, hypersensitivity, skin tests. 


\section{INTRODUÇÃO}

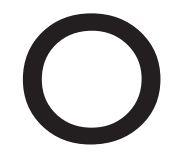
s corticosteroides (também designados glicocorticoides ou esteroides) (CT) são um grupo de hormonas esteroides produzidas pelas glândulas suprarrenais ou derivados sintéticos'. São compostos químicos derivados do colesterol, cuja estrutura consiste em três anéis com seis átomos de carbono e um anel com cinco átomos de carbono'. Os diferentes CT apresentam variações a nível das ligações covalentes, particularmente nos grupos metil e ligações acetonido, que determinam as suas características de solubilidade, preparação e degradação².

As propriedades terapêuticas dos CT foram demonstradas por Edward Kendall e Philip Hench em 19483,4. Consideram-se substâncias lipofílicas que interferem no metabolismo dos hidratos de carbono, lípidos e proteínas, sendo considerados uma das classes farmacológicas mais potentes com funções anti-inflamatórias, imunossupressoras, antiproliferativas e antialérgicas. O principal mecanismo de ação consiste na inibição da fosfolipase $A_{2}$ e consequente inibição do metabolismo do ácido araquidónico e outros mediadores inflamatórios, como prostaglandinas, tromboxanos e leucotrienos ${ }^{1,5,6}$. Durante a década de 1950 descobriu-se que a hidrocortisona poderia reduzir a inflamação e proliferação em algumas doenças de pele ${ }^{7}$. As modificações químicas deste fármaco deram origem a múltiplos CT de potência variável, cada um com propriedades específicas ${ }^{7}$.

Quanto às vias de administração, estão disponíveis formulações administradas por vias oral, intramuscular, endovenosa, intra-articular e tópica (nomeadamente: cutânea, nasal, inalatória e ocular) ${ }^{8}$.

A hipersensibilidade aos CT, com apresentação imediata ou tardia, tem sido descrita na literatura nos últimos 30 anos $^{9}$. O diagnóstico de uma reação de hipersensibilidade a CT continua a ser um desafio?

Com a presente revisão pretende-se caracterizar a epidemiologia, manifestações clínicas, abordagem diag- nóstica e orientação terapêutica perante reações de hipersensibilidade a CT.

\section{HIPERSENSIBILIDADE A CORTICOSTEROIDES}

\section{Epidemiologia}

Apesar de ser frequente o uso de CT em diferentes doenças, as reações de hipersensibilidade (HS) são incomuns, podendo ser subdiagnosticadas especialmente nos casos em que os CT são utilizados no tratamento de reações alérgicas ${ }^{10}$.

A incidência de reações de HS aos CT na população geral é baixa, com valores reportados na ordem dos $0,1-0,3 \%$ "I, sendo mais comuns em doentes sob terapêutica frequente com estes fármacos ${ }^{12}$. Os CT mais frequentemente implicados são a metilprednisolona e a hidrocortisona ${ }^{6}$.

Em termos epidemiológicos, as reações de hipersensibilidade do tipo imediato podem atingir todas as faixas etárias e ocorrem de forma semelhante em ambos os sexos".

\section{Via tópica}

A frequência de reações de HS a CT tópicos cutâneos varia de 0,2 a $5 \%{ }^{11,13}$. Habitualmente as reações são do tipo tardio, sendo a dermatite de contacto a manifestação clínica mais comum ${ }^{9-11}$.

\section{Via sistémica}

As reações de HS após administração sistémica de CT são raramente descritas na literatura ${ }^{7}$. Em 1998, Klein-Gitelman et al, num estudo de coorte de 213 crianças com doença reumatológica, verificou uma taxa de incidência de $0,1 \%$ de reações de HS, na sua maioria imediatas, num total de 10000 doses de CT sistémicos administradas ${ }^{7}$.

$\mathrm{Na}$ literatura estão descritos cerca de 100 casos de reações de hipersensibilidade imediata a CT após administração oral e parentérica ${ }^{14}$. 


\section{Vias de sensibilização}

Existe uma enorme variedade de moléculas de CT e muitas vias de administração disponíveis ${ }^{7}$. Terapêuticas tópicas e sistémicas são amplamente utilizadas, podendo qualquer uma delas levar a sensibilização do doente ${ }^{7}$. A via de sensibilização mais comum é, no entanto, a via tópica cutânea, sendo a sensibilização por via nasal ou brônquica menos frequente ${ }^{7}$.

É de realçar que um doente que apresente uma reação de HS a um CT administrado por via sistémica pode apresentar como via de sensibilização a via tópica, e vice-versa ${ }^{7}$.

\section{Fatores de risco}

\section{Via tópica}

Os doentes submetidos a tratamentos tópicos cutâneos de longo prazo, como no eczema crónico, dermatite de estase, ulceração crónica, dermatite actínica crónica, entre outras situações, apresentam um risco superior de sensibilização,15. A alteração da barreira cutânea e/ou ambiente pró-inflamatório presente nestas patologias são os fatores determinantes mais prováveis ${ }^{7}$.

Vários estudos referem a possibilidade de a atopia ser considerada fator de risco de sensibilização aos CT tó$\operatorname{picos}^{7,10,16,17}$.

\section{Via sistémica}

Os fatores de risco para o desenvolvimento de sensibilização aos CT sistémicos ainda não estão esclarecidos, contudo há descrição de uma maior ocorrência de reações em doentes asmáticos e em doentes tratados regularmente com CT sistémicos ${ }^{7}$.

\section{Hipersensibilidade alérgica imediata}

As reações de HS podem ser classificadas com base no intervalo de tempo entre a exposição ao fármaco e o início das manifestações clínicas $^{18}$. As reações imediatas ocorrem frequentemente com um intervalo de tempo inferior a uma hora e as reações tardias manifestam-se em intervalos de tempo variáveis, entre mais de uma hora a vários dias após a administração do fármaco ${ }^{18-20}$.

As reações do tipo imediato são habitualmente lgE mediadas; contudo, estas reações podem ser também dependentes da ativação de células $T$ com posterior libertação de mediadores pró-inflamatórios e citocinas ${ }^{18,19}$. Há diversas teorias sobre a ativação celular $T$ nas reações de hipersensibilidade do tipo imediato, sendo as mais amplamente aceites as seguintes:

- Teoria de ativação por haptenos: Consiste na ligação de aminoácidos resultantes do metabolismo do fármaco que interagem com o complexo principal de histocompatibilidade (MHC) e resultam na apresentação a células apresentadoras de antigénios (APC);

- Conceito p-i: Um fármaco quimicamente inerte, na sua forma nativa, liga-se diretamente (por ligações não covalentes) a moléculas pertencentes ao antígeno leucocitário humano (HLA) ou recetores de células T (TCR), induzindo a ativação e a proliferação clonal de linfócitos $T$, após contacto com a $\mathrm{APC}^{18,19}$.

Clinicamente, as reações imediatas apresentam um largo espetro de gravidade, de reações que cursam com manifestações mucocutâneas isoladas, como urticária e/ou angioedema, a reações de maior gravidade que se apresentam com anafilaxia/choque anafilático ${ }^{18,21,22}$. Os casos relatados de anafilaxia e fatalidades ocorreram na primeira hora após administração de $\mathrm{CT}^{\prime \prime}$.

As reações de HS do tipo imediato a CT são menos frequentes relativamente às reações HS tardias ${ }^{20,21}$.

Os CT sistémicos mais frequentemente implicados são a metilprednisolona (4l\%), a prednisolona (20\%), a triamcinolona (14\%) e a hidrocortisona (10\%) e a apresentação clínica mais frequentemente descrita na literatura é a urticária generalizada $8,11,20$. Contrariamente, a hipersensibilidade à dexametasona é extremamente $\operatorname{rara}^{20}$. 


\section{Hipersensibilidade alérgica tardia}

As reações tardias, também designadas por reações de HS do tipo IV, ocorrem através da estimulação de vários tipos de células T por antigénios específicos e atingem frequentemente a pele (um órgão rico em linfócitos $T$ e APC) $)^{18,22}$

Dependendo do tipo de citocinas produzidas pelos linfócitos $T$ e o tipo de células efetoras envolvidas, as reações de tipo IV subdividem-se em IVa (células ThI produtoras de IFN-y e TNF-a com ativação de monócitos/ /macrófagos), IVb (células Th2 produtoras de IL4/IL5/ILI3 com recrutamento e ativação de eosinófilos), IVc (linfócitos T CD4+ e CD8+ com capacidade citotóxica) e tipo IVd (linfócitos produtores de CXCL-8/GM-CSF com recrutamento de neutrófilos) ${ }^{18,22}$.

Clinicamente, as reações de HS tardias podem manifestar-se num espetro que pode variar de urticária, eritema pigmentado fixo, eritema multiforme (associado ou não a componente vasculítico), reação a fármacos com eosinofilia e sintomas sistémicos (DRESS), pustulose exantemática generalizada aguda (AGEP) e dermatoses boIhosas (síndrome de Stevens-Johnson / necrólise epidérmica tóxica) ${ }^{18,21,22}$.

As reações de hipersensibilidade tardias a CT têm, geralmente, uma apresentação clínica menos grave, consistindo sobretudo em manifestações de dermatite de contacto, por vezes associada a eczema e/ou angioedema ${ }^{6,13}$. A principal via de sensibilização aos CT é a pele ${ }^{6,13}$. Contudo, outras vias de sensibilização são possíveis, como o contacto indireto através da exposição a aerossóis ${ }^{13}$. Estão descritos na literatura casos de dermatite de contacto em funcionários da área da saúde e familiares de doentes com exposição frequente a aerossóis de budesonida ${ }^{13}$. Outras vias de sensibilização foram descritas, conjuntival, nasal, respiratória e gastrointestinal ${ }^{13}$.

O reconhecimento das reações de HS do tipo tardio a CT pode ser um desafio diagnóstico, dada a sua apresentação pouco específica, exigindo um elevado índice de suspeição clínica, particularmente no grupo de doen- tes que apresenta agravamento clínico da patologia cutânea após a sua utilização ${ }^{13}$. A apresentação clínica poderá associar-se ainda aos efeitos adversos "clássicos" da utilização crónica de CT, como a atrofia cutânea, a rosácea e a dermatite perioral ou perinasal. Em doentes sensibilizados, a utilização de CT tópicos de uso oftálmico pode resultar em edema facial ou periorbitário e/ou eczema, conjuntivite, enquanto os CT nasais/inalados podem provocar erupções eczematosas em torno dos orifícios (narinas e lábios), bem como reações na mucosa, como estomatite, congestão nasal e rinite crónica ${ }^{8,10,13,23}$. Estão descritas reações de flare-up em zonas de pele previamente afetada ${ }^{13}$.

Os excipientes utilizados nas preparações tópicas destes fármacos também podem causar dermatite de contacto e deverão ser testados em casos suspeitos ${ }^{9}$. Os componentes envolvidos podem ser ésteres (por exemplo succinato, fosfato, butirato e aceponato), lactose, parabenos, formaldeído, isotiazolinonas, lanolina, perfumes, propilenoglicol, carboximetilcelulose, álcool benzílico, polissorbato/tween 80, alergénios comummente presentes em preparações tópicas $9,10,14,24$. Estes aditivos resultam no aumento da penetração e/ou solubilidade do CT na pele 9 . O éster de sucinato, em particular, é utilizado para aumentar a solubilidade em preparações parentéricas e foi implicado como alergénio em vários casos descritos de hipersensibilidade imediata a $\mathrm{CT}{ }^{9}$.

\section{Hipersensibilidade não alérgica}

As reações de HS não alérgicas são mais frequentes, sendo também designadas por "pseudoalergias", "falsas alergias" ou "intolerância a fármacos" 25 . Estas reações podem ocorrer após a primeira administração do fármaco, não requerendo sensibilização prévia ${ }^{26}$. Um exemplo deste tipo de hipersensibilidade é a toxicidade cardiovascular aguda após a administração de CT em altas doses e/ou em perfusão rápida ${ }^{7}$. A apresentação clínica como broncospasmo pode ocorrer pela ação de inibição da enzima ciclo-oxigenase ${ }^{6}$ como no broncospasmo induzido por AINEs em doentes com asma ${ }^{6}$. 


\section{CLASSIFICAÇÃO, POTÊNCIA E REATIVIDADE CRUZADA}

A maioria dos estudos sobre alergia aos $\mathrm{CT}$ e reatividade cruzada referem-se aos $C T$ tópicos e baseiam-se nos resultados de testes epicutâneos ${ }^{7}$. Atualmente, perfis semelhantes de classificação são aplicados aos CT sistémicos ${ }^{7}$.

A classificação proposta inicialmente em 1989 por Coopman et al categoriza os CT tópicos em quatro grupos farmacológicos principais, com base nas características químicas e configurações moleculares: A, B, C e D (Quadro I) ${ }^{7,27,28}$, posteriormente reforçada pelos trabalhos de Lepoittevin et al ${ }^{7}$. Mais tarde, em 2000, Matura et al, através da análise do comportamento particular de certos constituintes dos ésteres do grupo $D$, propôs a subdivisão adicional em dois subgrupos, ou seja DI, constituído por ésteres estáveis, e D2, constituído por ésteres lábeis ${ }^{7,29}$. A labilidade destes ésteres condiciona uma alteração a nível das propriedades lipofílicas (aumentando a penetração na pele), resultando numa metabolização mais rápida ${ }^{7}$.

As reações de HS, baseadas em resultados de testes epicutâneos, têm sido descritas com maior frequência para os grupos que não apresentam metilação e halogenação no carbono 16 (grupos A e D2 e também com a budesonida) em comparação com os grupos $\mathrm{C}$ e DI7. O comportamento da budesonida, que não só reage com outros acetonidos, como também com ésteres, pode ser atribuído à sua estrutura molecular única e estereoespecificidade, resultando numa semelhança com moléculas dos grupos $B$ e $D^{7}$.

$A$ reatividade cruzada é frequente dentro de cada grupo $^{8}$, sendo também observada, em menor grau, entre os grupos $\mathrm{A}$ e $\mathrm{D} 2^{28}$. Os $\mathrm{CT}$ dos grupos $\mathrm{C}$ e DI raramente cursam com reações de $\mathrm{HS}$ e, geralmente, não interagem com CT de outros grupos ${ }^{7}$. Os CT que apresentam substituição no grupo metil $\mathrm{Cl} 6$ ou alteração do éster em CI7, como é o exemplo do furoato de mometasona e do propionato de fluticasona, apresentam caraterísticas próprias, impedindo a ligação proteína-hapteno, o que justifica o baixo risco de sensibilização primária a estes CT e o baixo risco de reatividade cruzada ${ }^{7}$.
Quadro I. Caracterização clássica de corticosteroides. A negrito apresenta-se um elemento representativo de cada grupo (Adaptado de ${ }^{29}$ ).

\begin{tabular}{|c|c|c|}
\hline \multicolumn{2}{|r|}{ Classes de corticosteroides } & \multirow[b]{2}{*}{$\begin{array}{l}\text { Notas } \\
\\
\end{array}$} \\
\hline $\mathbf{A}$ & $\begin{array}{l}\text { Hidrocortisona } \\
\text { Acetato de hidrocortisona } \\
\text { 2I- butirato de hidrocortisona } \\
\text { Hemisuccionato de hidrocortisona } \\
\text { Acetato de metilprednisolona } \\
\text { Hemisuccinato de } \\
\text { metilprednisolona } \\
\text { Prednisona } \\
\text { Prednisolona } \\
\text { Caproato de prednisolona } \\
\text { Acetato de prednisolona } \\
\text { Pivalato de prednisolona } \\
\text { Benzoato de prednisolona } \\
\text { Succinato de prednisolona } \\
\text { Acetato de fludrocortisona } \\
\text { Pivalato de tixocortol } \\
\text { Acetato de cortisona } \\
\text { Acetato de fludrocortisona } \\
\text { Fluorometolona } \\
\text { Acetato de fluprednisolona }\end{array}$ & \\
\hline B & $\begin{array}{l}\text { Acetonido de triamcinolona } \\
\text { Diacetato de triamcinolona } \\
\text { Triamcinolona } \\
\text { Hexacetonido de triamcinolona } \\
\text { Budesonida } \\
\text { Desonido } \\
\text { Fluocinonido } \\
\text { Halcinonido } \\
\text { Acetonido de fluocinolona }\end{array}$ & \\
\hline C & $\begin{array}{l}\text { Betametasona } \\
\text { Fosfato dissódico de betametasona } \\
\text { Dexametasona } \\
\text { Fosfato dissódico de dexametasona } \\
\text { Acetato de dexametasona } \\
\text { Valerato de diflucortolona } \\
\text { Desoximetasona } \\
\text { Fluocortolona } \\
\text { Pivalato de fluocortolona } \\
\text { Halometasona } \\
\text { Acetato de fluprednideno } \\
\text { Clocortolona }\end{array}$ & \\
\hline DI & $\begin{array}{l}\text { Dipropionato } \\
\text { de betametasona } \\
\text { Dipropionato de beclometasona } \\
\text { I } 7 \text {-valerato de betametasona } \\
\text { Propionato de clobetasol } \\
\text { Butirato de clobetasol } \\
\text { Diacetato de diflorasona } \\
\text { Propionato de fluticasona } \\
\text { Furoato de mometasona } \\
\text { Valerato de diflucortolona } \\
\text { I7,2I-dipropionato de } \\
\text { alclometasona }\end{array}$ & \\
\hline D2 & $\begin{array}{l}\text { Aceponato } \\
\text { de metilprednisolona } \\
\text { Valerato de hidrocortisona } \\
\text { Prednicarbato } \\
\text { I7-butirato de hidrocortisona } \\
\text { I7-butirato de hidrocortisona } \\
\text { I7-aceponato de hidrocortisona }\end{array}$ & $\begin{array}{l}\text { Pode causar } \\
\text { reatividade } \\
\text { cruzada com } \\
\text { budesonida e } \\
\text { corticoesteroides } \\
\text { do grupo A }\end{array}$ \\
\hline
\end{tabular}


Com base em resultados de testes epicutâneos e na análise molecular dos $\mathrm{CT}$, Baeck et al propuseram em 2011 uma classificação mais simplificada, que subdivide os CT em três grupos distintos (Quadro 2):
- Grupo I: Moléculas não metiladas e, maioritariamente, não halogenadas, anteriormente integradas nos grupos A, D2 e budesonida. Trata-se do grupo com maior ocorrência de reações alérgicas.

Quadro 2. Nova classificação de corticosteroides (Adaptado de ${ }^{13}$ )

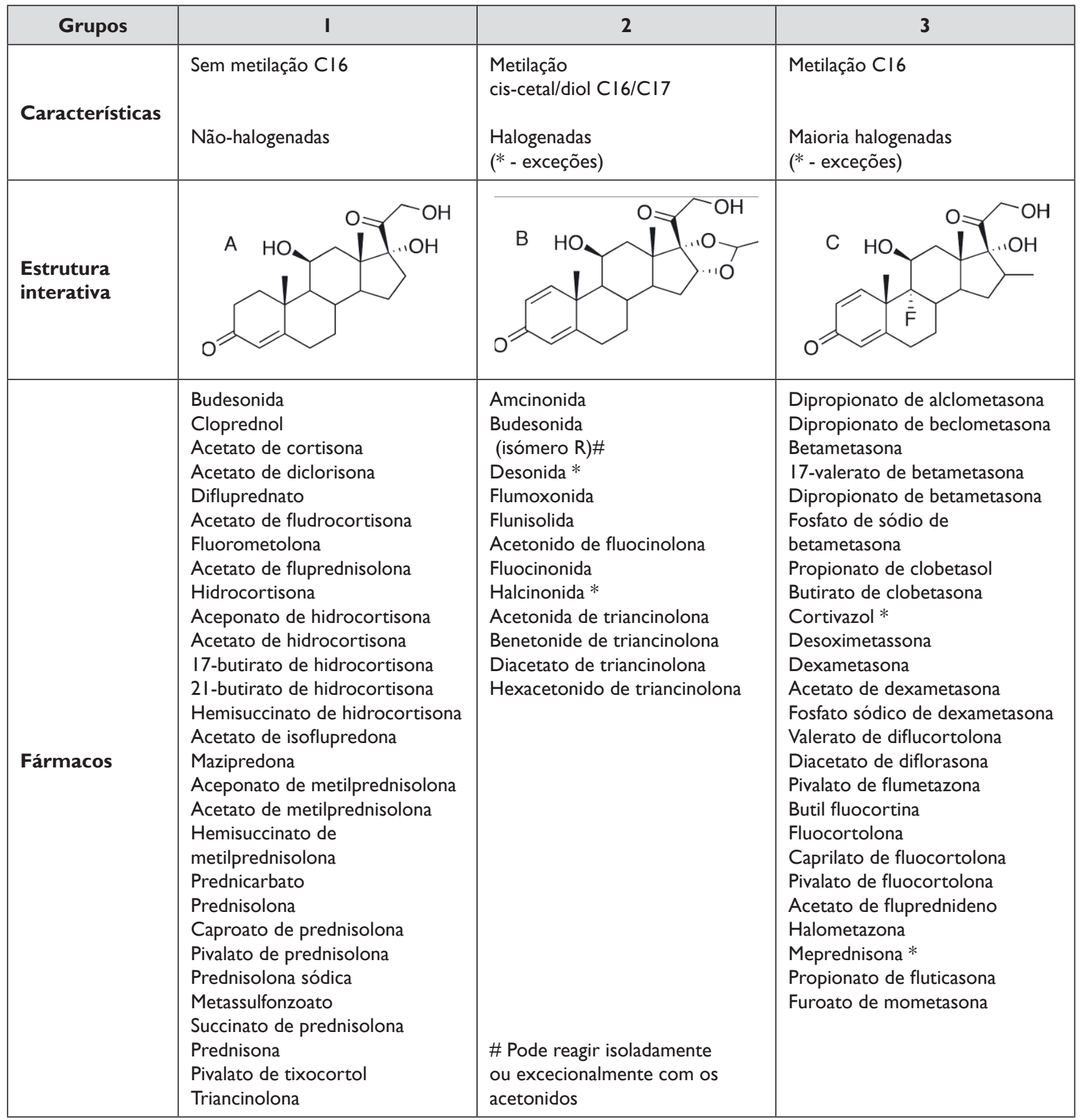


- Grupo 2: Moléculas halogenadas com estrutura cis-cetal/diol Cl6/Cl7; corresponde ao anterior grupo B.

- Grupo 3: Moléculas halogenadas e metiladas em $\mathrm{Cl6}$; corresponde aos anteriores grupos $\mathrm{C}$ e DI. Os CT deste grupo raramente cursam com reações de hipersensibilidade ${ }^{13}$.

Tendo em conta a nova classificação, foram identificados dois perfis de HS tardia aos CT tópicos: perfil I, em que os doentes reagem a moléculas de um único grupo (mais frequentemente do grupo I); e perfil 2, em que os doentes podem reagir a qualquer CT (isto é, Grupo I e Grupo 2 e/ou Grupo 3). Assim, para doentes com o perfil I, os agentes alternativos podem ser escolhidos dentro dos grupos 2 e $3^{13}$. Para os doentes correspondentes ao perfil 2, a utilização de um CT alternativo deverá ser individualizada, após avaliação de sensibilização/ /tolerância ${ }^{13,30}$. Outra opção alternativa aos CT tópicos, em algumas patologias cutâneas, são os inibidores da calcineurina, tacrolimus e pimecrolimus ${ }^{13}$.

A reatividade cruzada entre $C T$ foi também observada para as reações imediatas, no entanto com uma classificação e padrão de reatividade cruzada diferentes das reações tardias, mais associadas a CT tópicos ${ }^{13}$. Deste modo, estão descritos alguns casos de reações de hipersensibilidade à hidrocortisona e metilprednisolona sem evidência de reatividade cruzada ${ }^{13}$. Vários autores relataram reações alérgicas apenas aos CT associados a ésteres de succinato na sua composição, não apresentando assim reatividade cruzada com moléculas não esterificadas, ou com aquelas que apresentavam outros ésteres em substituição, isto é, fosfato ou acetato ${ }^{13}$. Na maioria dos casos de reação imediata a CT sistémicos, os doentes apresentam tolerância a moléculas halogenadas, como por exemplo à betametasona e à dexametasona ${ }^{13}$. A estratégia diagnóstica assenta na avaliação sistemática e individualizada dos perfis de sensibilização e tolerância ${ }^{13}$. Num estudo português foram estudados retrospetivamente seis doentes com história de reação imediata a um ou mais CT sistémicos (metilprednisolona, prednisolona e/ou hidrocortisona) ${ }^{20}$ submetidos a testes cutâneos por picada e intradérmicos com leitura imediata a hidrocortisona, metilprednisolona, prednisolona e dexametasona, apresentando todos eles resultados positivos para pelo menos um dos $\mathrm{CT}$ testados e em três doentes para mais do que um $\mathrm{CT}^{20}$. Todos os testes realizados com a dexametasona foram negativos, o que permitiu a sua utilização como CT alternativo por via parentérica em dois doentes ${ }^{20}$. Todos foram submetidos a prova de provocação oral alternativa com deflazacorte, apresentando resultados negativos em todos os casos avaliados, por isso este estudo sugere a utilização de deflazacorte como alternativa terapêutica viável e segura para administração oral em doentes com história de reação de HS a CT sistémicos ${ }^{20}$.

A potência dos $C T$ está estreitamente relacionada com a sua potência anti-inflamatória. O Quadro 3 apresenta a potência relativa e as doses equivalentes dos CT sistémicos e o Quadro 4 agrupa os CT tópicos por graus de potência ${ }^{23,31}$.

\section{DIAGNÓSTICO DE REAÇÕES DE HIPERSENSIBILIDADE A CORTICOESTEROIDES}

Os testes cutâneos são ferramentas diagnósticas de grande utilidade na avaliação da $\mathrm{HS}$ a fármacos ${ }^{9}$. A determinação sérica dos valores de lgE específica para diagnóstico de HS a CT não está disponível na prática clínica diária, sendo atualmente apenas realizada no âmbito de investigação científica".

O algoritmo para abordagem diagnóstica de doentes com história suspeita de reações de HS após administração de CT depende da via de administração utilizada (tópica ou sistémica) e do tempo decorrido entre a administração do medicamento e o desenvolvimento de sintomas $^{10}$. Na Figura I apresentamos uma proposta de algoritmo de abordagem às reações de HS a CT. 
Quadro 3. Potências relativas e doses equivalentes de corticosteroides (Adaptado de ${ }^{31}$ )

\begin{tabular}{|l|c|c|c|}
\hline \multicolumn{1}{|c|}{ CT TÓPICOS } & $\begin{array}{c}\text { Dose equivalente de } \\
\text { glucocorticoide } \\
\text { (mg) }\end{array}$ & $\begin{array}{c}\text { Potência relativa à } \\
\text { hidrocortisona (cortisol): } \\
\text { Atividade anti-inflamatória }\end{array}$ & $\begin{array}{c}\text { Potência relativa à } \\
\text { hidrocortisona (cortisol): } \\
\text { Atividade } \\
\text { mineralocorticoide }\end{array}$ \\
\hline $\begin{array}{l}\text { Ação rápida } \\
\text { Hidrocortisona } \\
\text { Acetato de cortisona }\end{array}$ & 20 & 1 & 1 \\
\hline $\begin{array}{l}\text { Ação imediata } \\
\text { Prednisona }\end{array}$ & 25 & 0,8 & 0,8 \\
$\begin{array}{l}\text { Prednisolona } \\
\text { Triancinolona }\end{array} \quad$ Metilprednisolona & 5 & 4 & 0,8 \\
\hline $\begin{array}{l}\text { Ação longa } \\
\text { Dexametasona } \\
\text { Betametasona }\end{array}$ & 5 & 4 & 0,8 \\
\hline $\begin{array}{l}\text { Ação } \\
\text { mineralocorticoide } \\
\text { Fludrocortisona }\end{array}$ & 4 & 5 & Não aplicável \\
Aldosterona & 0,75 & 30 & 0,5 \\
\hline
\end{tabular}

Quadro 4. Subdivisão em grupos de corticosteroides tópicos por potência (Adaptado de ${ }^{23}$ )

\begin{tabular}{|l|l|}
\hline $\begin{array}{l}\text { Corticosteroides } \\
\text { de potência } \\
\text { muito alta }\end{array}$ & $\begin{array}{l}\text { Dipropionato de betametasona 0,05\% } \\
\text { Propionato de clobetasol 0,05\% } \\
\text { Diacetato de diflorasona 0,05\% } \\
\text { Fluocinonido 0,1\% } \\
\text { Propionato de halobetasol 0,05\% }\end{array}$ \\
\hline $\begin{array}{l}\text { Corticosteroides } \\
\text { de alta potência }\end{array}$ & $\begin{array}{l}\text { Amcinonido 0,1\% } \\
\text { Dipropionato de betametasona 0,05\% }\end{array}$ \\
\hline $\begin{array}{l}\text { Corticosteroides } \\
\text { de potência } \\
\text { média a alta }\end{array}$ & $\begin{array}{l}\text { Desoximetasona } \\
\text { Diacetato de diflorasona 0,05\% } \\
\text { Fluocinonido } \\
\text { Halcinonido 0, I\% } \\
\text { Dipropionato de betametasona 0,05\% } \\
\text { Propionato de fluticasona 0,005\% } \\
\text { Acetonido de triamcinolona a 0,5\% }\end{array}$ \\
\hline
\end{tabular}

\section{Via tópica}

Relativamente às reações de hipersensibilidade a CT tópicos, o gold-standard para o diagnóstico são os testes epicutâneos/patch tests, que permitem uma deteção de cerca de $90 \%$ dos doentes alérgicos ${ }^{10,32}$ - Quadro 5 . Os resultados falsos positivos podem resultar de reações cutâneas irritativas de caráter não alérgico ${ }^{10}$.
Atualmente o pivalato de tixocortol e a budesonida pertencem às baterias de testes epicutâneos-padrão americana e europeia Thin-layer rapid use epicutaneous patch test for Topical Use Only (T.R.U.E. test) e The European Baseline Series, respetivamente ${ }^{33}$. Num trabalho realizado em Inglaterra foi estudada uma população de 2123 doentes com dermatite de contacto a CT e aplicados testes epicutâneos com seis CT diferentes - pivalato de tixocortol, budesonida, butirato de hidrocortisona, valerato de betametasona, butirato de clobetasona e propionato de clobetasol ${ }^{34}$. A combinação dos resultados dos testes epicutâneos ao pivalato de tixocortol e budesonida permitiu a identificação de cerca de $91,3 \%$ dos doentes desta população" ${ }^{11,34}$. Num estudo retrospetivo realizado nos EUA foram avaliados os resultados de testes epicutâneos em doentes com suspeita de dermatite de contacto a CT tópicos num período de 6 anos $^{33}$. O pivalato de tixocortol e a budesonida têm sido dos fármacos mais frequentemente implicados nas reações de HS a CT tópicos, sob forma de apresentação de dermatite de contacto ${ }^{13,33}$.

Apesar de os testes epicutâneos serem recomendados como método de eleição para o diagnóstico de 


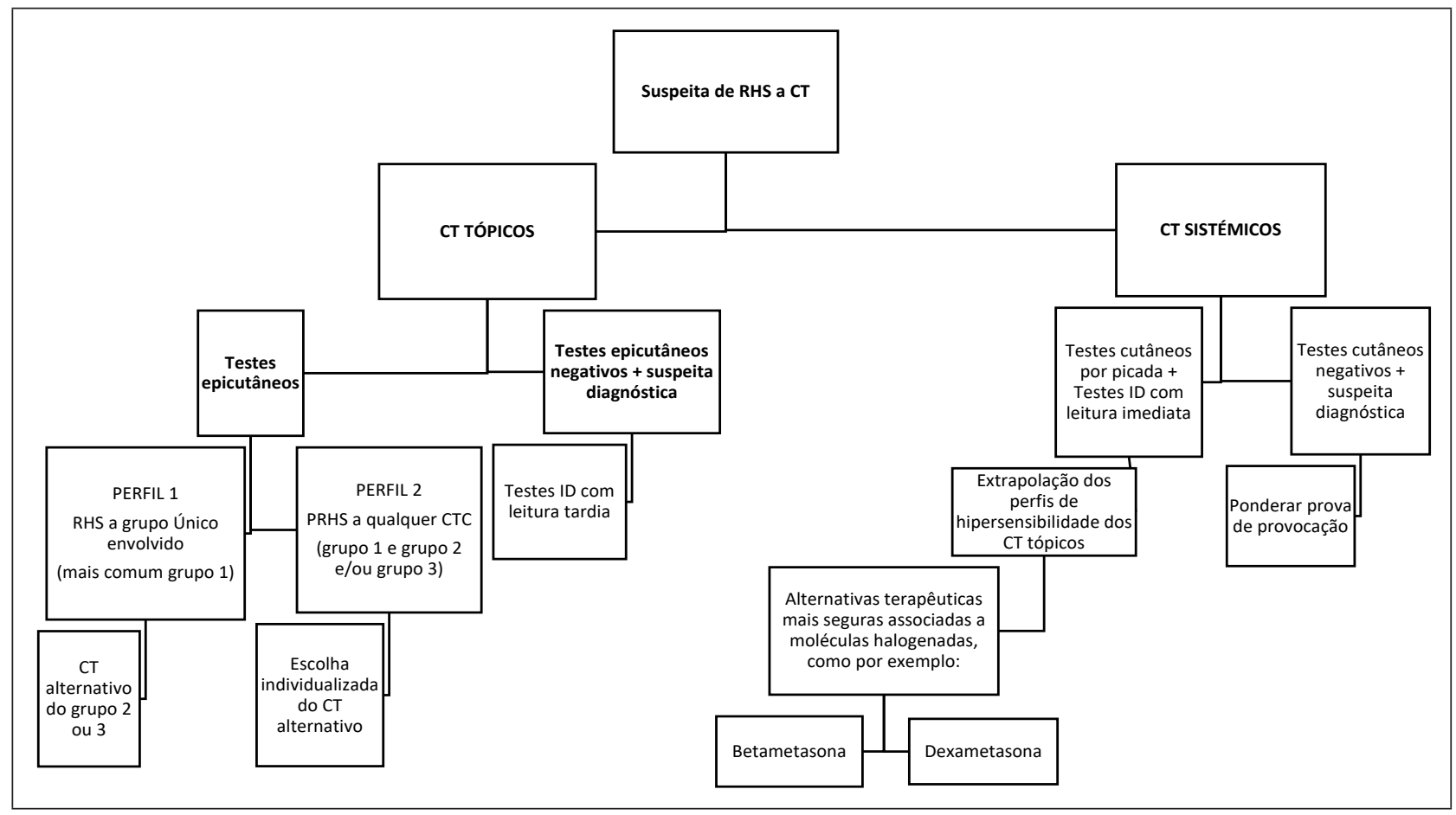

CT - Corticoesteroides; ID - Intradérmicos; RHS - Reações de Hipersensibilidade

Figura I. Algoritmo de abordagem das reações de hipersensibilidade a corticoesteroides.

Quadro 5. Concentrações de corticosteroides e seus excipientes para realização de testes cutâneos por picada e intradérmicos $9,14,40$

\begin{tabular}{|c|c|c|c|c|}
\hline \multirow{2}{*}{ Corticosteroides } & \multirow{2}{*}{$\begin{array}{l}\text { Testes cutâneos } \\
\text { por picada }\end{array}$} & \multicolumn{3}{|c|}{ Testes cutâneos intradérmicos } \\
\hline & & $1 / 1000$ & $1 / 100$ & $1 / 10$ \\
\hline $\begin{array}{l}\text { Metilprednisolona } \\
\text { Hidrocortisona } \\
\text { Prednisolona } \\
\text { Betametasona } \\
\text { Dexametasona } \\
\text { Budesonida } \\
\text { Triamcinolona } \\
\text { Prednisona } \\
\text { Deflazacorte }\end{array}$ & $\begin{array}{c}4 \text { ou } 40 \mathrm{mg} / \mathrm{mL} \\
100 \mathrm{mg} / \mathrm{mL} \\
10 \text { a } 30 \mathrm{mg} / \mathrm{mL} \\
4 \mathrm{mg} / \mathrm{mL} \\
4 \mathrm{mg} / \mathrm{mL} \\
0,25 \mathrm{mg} / \mathrm{mL} \\
40 \mathrm{mg} / \mathrm{mL} \\
30 \mathrm{mg} / \mathrm{mL} \\
30 \mathrm{mg} / \mathrm{mL}\end{array}$ & $\begin{array}{c}0,04 \mathrm{mg} / \mathrm{mL} \\
0,1 \mathrm{mg} / \mathrm{mL} \\
0,1 \mathrm{mg} / \mathrm{mL} \\
0,004 \mathrm{mg} / \mathrm{mL} \\
0,004 \mathrm{mg} / \mathrm{mL} \\
0,00025 \mathrm{mg} / \mathrm{mL} \\
0,004 \mathrm{mg} / \mathrm{mL} \\
- \\
-\end{array}$ & $\begin{array}{c}0,4 \mathrm{mg} / \mathrm{mL} \\
\mathrm{Img} / \mathrm{mL} \\
0,1 \mathrm{mg} / \mathrm{mL} \\
0,04 \mathrm{mg} / \mathrm{mL} \\
0,04 \mathrm{mg} / \mathrm{mL} \\
0,0025 \mathrm{mg} / \mathrm{mL} \\
0,04 \mathrm{mg} / \mathrm{mL} \\
- \\
-\end{array}$ & $\begin{array}{c}4 \mathrm{mg} / \mathrm{mL} \\
10 \mathrm{mg} / \mathrm{mL} \\
- \\
0,4 \mathrm{mg} / \mathrm{mL} \\
0,4 \mathrm{mg} / \mathrm{mL} \\
0,025 \mathrm{mg} / \mathrm{mL} \\
0,4 \mathrm{mg} / \mathrm{mL} \\
- \\
-\end{array}$ \\
\hline \multicolumn{5}{|c|}{ Excipientes } \\
\hline $\begin{array}{l}\text { Carboximetilcelulose } \\
\text { Álcool benzílico } \\
\text { Polissorbato/tween } \mathbf{8 0}\end{array}$ & $\begin{array}{c}5 \mathrm{mg} / \mathrm{mL} \\
10 \mathrm{mg} / \mathrm{mL} \\
0,4 \mathrm{mg} / \mathrm{mL}\end{array}$ & $\begin{array}{c}0,005 \mathrm{mg} / \mathrm{mL} \\
0,1 \mathrm{mg} / \mathrm{mL} \\
0,004 \mathrm{mg} / \mathrm{mL}\end{array}$ & $\begin{array}{c}0,05 \mathrm{mg} / \mathrm{mL} \\
\mathrm{Img} / \mathrm{mL} \\
0,04 \mathrm{mg} / \mathrm{mL}\end{array}$ & $\begin{array}{l}- \\
- \\
-\end{array}$ \\
\hline
\end{tabular}

reações de HS tardia a CT, a frequência de falso-negativos não é conhecida ${ }^{7,10,30}$ e alguns autores recomendam que, perante a suspeita diagnóstica e resultados negativos nos testes epicutâneos, sejam realizados testes intradérmicos com leituras tardias $(24-72 h)^{7,10,30}$.

Os testes intradérmicos com leituras tardias, apesar de detetarem casos adicionais de HS a CT, não devem 
ser realizados rotineiramente devido ao risco importante de atrofia cutânea ${ }^{30}$.

Deve ainda ter-se em consideração a possibilidade de hipersensibilidade aos excipientes em doentes com testes epicutâneos negativos aos CT apesar de clínica sugestiva, ou em doentes que toleram apenas formulações específicas ${ }^{9,14}$.

Os doentes com manifestações de dermatite de contacto a CT podem tolerar os mesmos fármacos administrados por via sistémica; no entanto, deve considerar-se a possibilidade de ocorrência de dermatite de contacto sistémica $^{10}$. Apesar de tipicamente as manifestações sistémicas de HS a CT se associarem a CT administrados por via sistémica, reações como dermatite de contacto sistémica podem ocorrer, nomeadamente após a aplicação de $\mathrm{CT}$ tópicos por via nasal ou inalatória ${ }^{7}$.

\section{Via sistémica}

Tipicamente as reações de HS imediata a CT associam-se a CT sistémicos administrados por via oral ou parentérica ${ }^{7,14}$. A abordagem diagnóstica inicial cursa com a realização de testes cutâneos por picada ${ }^{7,14}$ e testes intradérmicos com leitura imediata realizados em concentrações crescentes e não irritativas (Quadro 6) ${ }^{7,14}$.

Quadro 6. Corticoesteroides utilizados nos testes epicutâneos ${ }^{32}$

\section{Corticoesteroides - testes epicutâneos/patch-test}

Budesonida a $0,01 \%$ em parafina

I7-valerato de betametasona $1 \%$ em parafina

Acetonido de triamcinolona $1 \%$ em parafina

21 -pivalato de tixocortol $0,1 \%$ em parafina

17,21-dipropionato de alclometasona I\% em parafina

17-propionato de clobetasol- I\% em parafina

21 -fosfato dissódico de dexametasona $1 \%$ em parafina

Prednisolona $1 \%$ em parafina

Dexametasona $1 \%$ em absoluto ou em álcool

Betametasona $1 \%$ em absoluto ou em álcool

Hidrocortisona $1 \%$ em parafina

Prednisona $1 \%$ em absoluto ou em álcool

Acetato de hidrocortisona $1 \%$ em sulfóxido de dimetilo ou em álcool

Budesonida 0, $1 \%$ em parafina

I7-butirato de hidrocortisona $1 \%$ em álcool
Os testes podem também permitir a identificação de um agente alternativo ${ }^{14}$.

Dados recentes têm contribuído para aferir a validade da utilização dos testes intradérmicos no diagnóstico de reações de HS imediata a CT, contudo os valores de especificidade e de sensibilidade ainda não foram determinados 9,14 .

Apesar da baixa utilidade dos testes in vitro (IgE específica e teste de ativação de basófilos) no diagnóstico de HS a CT, foram descritos recentemente três casos de reações de HS imediata após administração de succinato de metilprednisolona, onde o mecanismo IgE mediado específico foi detetado, não só através da realização de testes cutâneos, como também através de testes in vitro, nomeadamente teste de ativação de basófilos e Immuno$C A P^{\circledR 35}$. O teste de ativação de basófilos consiste na identificação, por citometria de fluxo, do aumento da expressão de marcadores de superfície dos basófilos, após estimulação com alergénios ou fármacos ${ }^{36}$. $O$ Immuno$C A P^{\circledR}$ consiste num método de medição capaz de identificar e determinar semiquantitativamente a presença de anticorpos específicos da classe $\lg \mathrm{E}^{37}$.

Contudo, a prova de provocação duplamente cega e controlada por placebo mantém-se o gold standard para o diagnóstico ${ }^{5,8}$.

\section{DISCUSSÃO E CONCLUSÃO}

Os CT são uma classe farmacológica amplamente utilizada na prática clínica diária em diversos campos da medicina ${ }^{38}$, cujas reações de hipersensibilidade têm vindo a ser descritas na literatura com uma frequência crescente ${ }^{39}$.

A utilização de CT no tratamento de patologias alérgicas torna o diagnóstico de hipersensibilidade aos CT um desafio. A realização de uma história clínica detalhada associada a um elevado grau de suspeição são essenciais para o diagnóstico, sendo particularmente importante perante quadros de dermatite crónica recorrente, com 
uma evolução paradoxal pela possibilidade de sensibilização aos CT tópicos utilizados.

As reações do tipo imediato, apesar de menos frequentes, podem ser fatais e não devem ser subestimadas.

A realização de testes cutâneos por picada/intradérmicos e epicutâneos auxiliam na identificação do fármaco implicado e de potenciais alternativos. No entanto, a prova de provocação mantém-se o gold standard.

A investigação futura deverá aferir a utilidade dos testes cutâneos, de forma a elevar a capacidade de diagnóstico de hipersensibilidade aos CT e excipientes.

\section{Conflito de interesses}

Os autores declaram que não existem conflitos de interesses.

\section{Contacto:}

Iolanda Alen Coutinho

Serviço de Imunoalergologia, Centro Hospitalar

e Universitário de Coimbra

Praceta Prof. Mota Pinto

3000-075 Coimbra, Portugal

Email: iolandaalen@gmail.com

\section{REFERÊNCIAS}

I. Adcock IM, Mumby S. Glucocorticoids. Handb Exp Pharmacol 2017;237:171-96.

2. Berg JM, Tymoczko JL, Stryer L. Biochemistry. 5th edition. New York: W H Freeman; 2002. Available from: https://www.ncbi.nlm. nih.gov/books/NBK2II54/

3. Glyn JH. The discovery of cortisone: a personal memory. BMJ 1998;317(7161):822A.

4. Suji Eapen M, Dhar Shukla S, Quasir Mahmood M, McAlinden-Volkovickas K, D. Eri R, Haydn Walters E, et al. Corticosteroids and Steroids Therapy - New Research; 2015.

5. Yoshikawa, H., Tasaka, K. Anti-allergic action of glucocorticoids: Comparison with immunosuppressive and anti-inflammatory effects. Curr Med Chem Anti Inflamm Anti Allergy Agents; 2003. 2(I), 37-50.

6. Kamm GL, Hagmeyer KO. Allergic-type reactions to corticosteroids. Ann Pharmacother 1999;33(4):45I-60.

7. Baeck M, Marot L, Nicolas JF, Pilette C, Tennstedt D, Goossens A. Allergic hypersensitivity to topical and systemic corticosteroids: a review. Allergy 2009;64(7):978-94.
8. Ramirez R, Brancaccio RR. Reações cutâneas alérgicas a corticoides sistêmicos. 2007;82(2):169-76.

9. Baker A, Empson M, The R, Fitzharris P. Skin testing for immediate hypersensitivity to corticosteroids: a case series and literature review. Clin Exp Allergy 2015;45(3):669-76.

10. Torres MJ, Canto G. Hypersensitivity reactions to corticosteroids. Curr Opin Allergy Clin Immunol 2010;10(4):273-9.

II. Otani IM, Banerji A. Immediate and delayed hypersensitivity reactions to corticosteroids: Evaluation and management. Curr Allergy Asthma Rep 2016;16(3):18.

12. Vatti RR, Ali F, Teuber S, Chang C, Gershwin ME. Hypersensitivity reactions to corticosteroids. Clin Rev Allergy Immunol 2014;47(I):26-37.

13. Baeck M, Goossens A. Immediate and delayed allergic hypersensitivity to corticosteroids: practical guidelines. Contact Dermatitis 2012;66(I):38-45.

14. Venturini M, Lobera T, del Pozo MD, González I, Blasco A. Immediate hypersensitivity to corticosteroids. J Investig Allergol Clin Immunol 2006;16(I):5I-6.

15. Santos A, Andrade P, Loureiro C, Gonçalo M. Testes epicutâneos a corticosteróides num período de dez anos. Rev Port Imunoalergologia 201।;19(2):85-9I.

16. Saito R, Moroi S, Okuno H, Ogawa O. Anaphylaxis following administration of intravenous methylprednisolone sodium succinate in a renal transplant recipient. Int J Urol 2004;II(3): I7I-4.

17. Kilpiö K, Hannuksela M. Corticosteroid allergy in asthma. Allergy 2003;58(II):II3I-5.

18. Regateiro F, Faria E. Mecanismos imunopatológicos das reações de hipersensibilidade a fármacos. Rev Port Imunoalergologia 2016;24(2):63-78.

19. Stone SF, Phillips EJ, Wiese MD, Heddle RJ, Brown SG. Immediate-type hypersensitivity drug reactions. Br J Clin Pharmacol 2014;78(I):I-I3.

20. Sousa NG, Faria E, Carrapatoso I, Almeida E, Geraldes L, Chieira C. Deflazacort: a possible alternative in corticosteroid allergy. J Investig Allergol Clin Immunol 2010;20(5):449-5I.

2I. Burgdorff T, Venemalm L, Vogt T, Landthaler M, Stolz W. IgE-mediated anaphylactic reaction induced by succinate ester of methylprednisolone. Ann Allergy Asthma Immunol 2002;89(4):425-8.

22. Bircher AJ. Drug hypersensitivity. Chem Immunol Allergy 2014;100:120-31.

23. Ference JD, Last AR. Choosing topical corticosteroids. Am Fam Physician 2009;79(2):135-40.

24. Ericson-Neilsen W, Kaye AD. Steroids: pharmacology, complications, and practice delivery issues. Ochsner J 20I4;I4(2):203-7.

25. Tanno LK, Calderon MA, Smith HE, et al. Dissemination of definitions and concepts of allergic and hypersensitivity conditions. World Allergy Organ J 2016;9:24.

26. O'Hehir, R.E., Holgate, S.T., Sheikh, A. (20I7). Middleton's allergy essentials. 
27. Lepoittevin JP, Drieghe J, Dooms-Goossens A. Studies in patients with corticosteroid contact allergy. Understanding cross-reactivity among different steroids. Arch Dermatol 1995;13I(I):31-7.

28. Schellenberg R, D R Adachi J, Bowie D, Brown J, Guenther L, Kader $T$ et al. Oral corticosteroids in asthma : A review of benefits and risks. Can Respir J 2007;14(Suppl C):IC-7C.

29. Baeck M, Chemelle JA, Goossens A, Nicolas JF, Terreux R. Corticosteroid cross-reactivity: clinical and molecular modelling tools. Allergy 2011;66(10):1367-74.

30. Soria A, Baeck M, Goossens A, Marot L, Duveille V, Derouaux AS, et al. Patch, prick or intradermal tests to detect delayed hypersensitivity to corticosteroids?. Contact Dermatitis 2011;64(6):313-24.

31. Parente L. Deflazacort: therapeutic index, relative potency and equivalent doses versus other corticosteroids. BMC Pharmacol Toxicol 2017;18(I):I.

32. Mimesh S, Pratt M. Allergic contact dermatitis from corticosteroids: reproducibility of patch testing and correlation with intradermal testing. Dermatitis 2006;17(3):137-42.

33. Saripalli YV, Achen F, Belsito DV. The detection of clinically rel- evant contact allergens using a standard screening tray of twenty-three allergens. J Am Acad Dermatol 2003;49(I):65-9.

34. Boffa MJ, Wilkinson SM, Beck MH. Screening for corticosteroid contact hypersensitivity. Contact Dermatitis 1995;33(3):149-5I

35. Steiner M, Harrer A, Himly M. Basophil reactivity as biomarker in immediate drug hypersensitivity reactions-potential and limitations. Front Pharmacol 2016;7:171.

36. Carrapatoso I, Cadinha S, Sanz ML. Aplicação do teste de activação dos basófilos no estudo de reacções de hipersensibilidade a alimentos e fármacos. Rev Port Imunoalergol 2005; 13(2):153-64

37. van Hage M, Hamsten C, Valenta R. ImmunoCAP assays: Pros and cons in allergology. J Allergy Clin Immunol 2017;140(4):974-7.

38. Swartz SL, Dluhy RG. Corticosteroids: clinical pharmacology and therapeutic use. Drugs 1978;16(3):238-55.

39. Knarborg M, Bendstrup E, Hilberg $O$. Increasing awareness of corticosteroid hypersensitivity reactions is important. Respirol Case Rep 2013;I(2):43-5.

40. Vervloet D, Pradal M, Birnbaum J, Koeppel MC. Drug allergy. Paris. Phadia 201I;28(4):400-9. 